\title{
A NOTE ON AN OSCILLATION CRITERION FOR AN EQUATION WITH DAMPED TERM
}

\author{
JURANG YAN
}

\begin{abstract}
A new oscillation criterion is given for the equation $x^{\prime \prime}(t)+p(t) x^{\prime}(t)+$ $q(t) x(t)=0, t \in\left[t_{0}, \infty\right)$, where $p(t)$ and $q(t)$ are allowed to change sign on $\left[t_{0}, \infty\right)$.
\end{abstract}

Let us consider the second order differential equation with damped term

$$
x^{\prime \prime}(t)+p(t) x^{\prime}(t)+q(t) x(t)=0,
$$

and the more general equation

$$
x^{\prime \prime}(t)+p(t) x^{\prime}(t)+q(t) f(x(t))=0,
$$

where $p, q \in C\left[t_{0}, \infty\right)$ and are allowed to assume negative values for arbitrarily large $t, f \in C(R), x f(x)>0$ for $x \neq 0$.

We shall restrict our attention to solutions of (1) or (1)' which exist on some ray $[\tilde{t}, \infty)$. A solution of an equation is called oscillatory if it has no largest zero; otherwise it is nonoscillatory. An equation is said to be oscillatory if every solution is oscillatory.

For the second order linear differential equation

$$
x^{\prime \prime}(t)+q(t) x(t)=0,
$$

Wintner [6] proved that a sufficient condition for oscillation was

$$
\lim _{t \rightarrow \infty} \frac{1}{t} \int_{t_{0}}^{t} \int_{t_{0}}^{s} q(\tau) d \tau d s=\infty
$$

Hartman [3] proved that the limit cannot be replaced by the upper limit in condition $(* *)$ and

$$
-\infty<\lim _{t \rightarrow \infty} \inf \frac{1}{t} \int_{t_{0}}^{t} \int_{t_{0}}^{s} q(\tau) d \tau d s<\lim _{t \rightarrow \infty} \sup \frac{1}{t} \int_{t_{0}}^{t} \int_{t_{0}}^{s} q(\tau) d \tau d s \leqslant \infty
$$

implies $(*)$ is oscillatory.

Later, important developments by Willett and Coles in averaging techniques for oscillation of $(*)$ were made. Willett [5] and Coles [2], respectively, established more general theorems by considering weighted averages of the integral of $q$.

Received by the editors October 25, 1982.

1980 Mathematics Subject Classification. Primary 34C10, 34C15.

Key words and phrases. Second order differential equation with damped term, oscillation. 
Several years ago Kamenev [4] obtained an oscillation criterion for $(*)$, namely, $(*)$ is oscillatory if for some $n>2$,

$$
\lim _{t \rightarrow \infty} \sup t^{1-n} \int_{t_{0}}^{t}(t-s)^{n-1} q(s) d s=\infty
$$

which extended Wintner's result.

Recently, Yeh [8] has shown some oscillation criteria of (1)' by using a technique similar to Kamenev's, which included results of [1,4 and 6].

The purpose of this note is to proceed further in this direction and present a new oscillation theorem which improves Kamenev's criterion. A more general version of the theorem contains the theorems of Yeh [7 and 8].

Our result is as follows:

THEOREM. Suppose for some $\alpha \in(1, \infty)$ and $\beta \in[0,1)$,

$$
\lim _{t \rightarrow \infty} \sup t^{-\alpha} \int_{t_{0}}^{t}(t-s)^{\alpha} s^{\beta} q(s) d s=\infty,
$$

(3) $\lim _{t \rightarrow \infty} \sup t^{-\alpha} \int_{t_{0}}^{t}[(t-s) p(s) s+\alpha s-\beta(t-s)]^{2}(t-s)^{\alpha-2} s^{\beta-2} d s<\infty$.

Then (1) is oscillatory.

Proof. Assume the contrary. Then (1) has a nonoscillatory solution $x(t)$. Without loss of generality, we may assume $x(t) \neq 0$ for $t \geqslant t_{0}$. Define $\omega(t)=x^{\prime}(t) / x(t)$. Then it follows from (1) that

$$
\omega^{\prime}(t)+\omega^{2}(t)+p(t) \omega(t)+q(t)=0 .
$$

Hence

$$
\begin{aligned}
\int_{t_{0}}^{t}(t-s)^{\alpha} s^{\beta} \omega^{\prime}(s) d s & +\int_{t_{0}}^{t}(t-s)^{\alpha} s^{\beta} \omega^{2}(s) d s \\
& +\int_{t_{0}}^{t}(t-s)^{\alpha} s^{\beta} p(s) \omega(s) d s+\int_{t_{0}}^{t}(t-s)^{\alpha} s^{\beta} q(s) d s \leqslant 0
\end{aligned}
$$

Noting that

$$
\begin{aligned}
\int_{t_{0}}^{t}(t-s)^{\alpha} s^{\beta} \omega^{\prime}(s) d s= & \alpha \int_{t_{0}}^{t}(t-s)^{\alpha-1} s^{\beta} \omega(s) d s-\beta \int_{t_{0}}^{t}(t-s)^{\alpha} s^{\beta-1} \omega(s) d s \\
& -\omega\left(t_{0}\right)\left(t-t_{0}\right)^{\alpha} t_{0}^{\beta},
\end{aligned}
$$

we obtain

$$
\begin{aligned}
\int_{t_{0}}^{t}(t-s)^{\alpha} s^{\beta} q(s) d s \leqslant & \omega\left(t_{0}\right)\left(t-t_{0}\right)^{\alpha} t_{0}^{\beta}-\int_{t_{0}}^{t}(t-s)^{\alpha} s^{\beta} \omega^{2}(s) d s \\
& -\int_{t_{0}}^{t}[(t-s) p(s) s+\alpha s-\beta(t-s)](t-s)^{\alpha-1} s^{\beta-1} \omega(s) d s .
\end{aligned}
$$


Dividing by $t^{\alpha}$ and taking the upper limit as $t \rightarrow \infty$, we get

$$
\begin{aligned}
& \lim _{t \rightarrow \infty} \sup _{t^{-\alpha}} \int_{t_{0}}^{t}(t-s)^{\alpha} s^{\beta} q(s) d s \\
& \leqslant \omega\left(t_{0}\right) t_{0}^{\beta}+\lim _{t \rightarrow \infty} \sup \frac{t^{-\alpha}}{4} \int_{t_{0}}^{t}[(t-s) s p(s)+\alpha s-\beta(t-s)]^{2}(t-s)^{\alpha-2} s^{\beta-2} d s \\
& \quad-\lim _{t \rightarrow \infty} \inf t^{-\alpha} \int_{t_{0}}^{t}\left\{(t-s)^{\alpha / 2} s^{\beta / 2} \omega(s)\right. \\
& \left.\quad+\frac{1}{2}[(t-s) s p(s)+\alpha s-\beta(t-s)](t-s)^{(\alpha-2) / 2} s^{(\beta-2) / 2}\right\}^{2} d s<\infty,
\end{aligned}
$$

which contradicts conditions (2) and (3). This completes the proof.

Let $p(t)=0$. Then (3) is satisfied automatically. Thus we have

Corollary 1. Suppose for some $\alpha \in(1, \infty)$ and $\beta \in[0,1)$, (2) is satisfied. Then (1) is oscillatory.

REMARK 1. Corollary 1 improves and generalizes Kamenev's theorem [4].

From the proof of the theorem, we easily obtain the following extension to (1)'.

COROLlary 2. Suppose

$$
f^{\prime}(x) \text { exists and } f^{\prime}(x) \geqslant k>0
$$

for some constant $k$ and for all $x \neq 0$. If (2) and (3) hold, then (1)' is oscillatory.

Taking $\alpha=n-1, \beta=0$ in (2) and (3), we get

Corollary 3. Suppose (4) is satisfied. If

$$
\lim _{t \rightarrow \infty} \sup t^{1-n} \int_{t_{0}}^{t}(t-s)^{n-1} q(s) d s=\infty
$$

and

$$
\lim _{t \rightarrow \infty} \sup t^{1-n} \int_{t_{0}}^{t}[(t-s) p(s)+(n-1)]^{2}(t-s)^{n-3} d s<\infty
$$

for some $n>2$ (not necessarily integral), then (1)' is oscillatory.

Remark 2. Corollary 3 includes Kamenev's [4] and Yeh's theorem [7 and 8].

As an example, the equation

$$
x^{\prime \prime}(t)+\frac{\sin t}{t^{\mu}} x^{\prime}(t)+\frac{\cos t}{t^{\nu}} x(t)=0, \quad 1 \leqslant \mu<\infty, 0 \leqslant \nu<1 .
$$

Taking $\alpha=2, \nu<\beta<1$, we easily verify that all conditions of our theorem are satisfied. Hence, (5) is oscillatory. However, each of the criteria in $[4,7$ and 8$]$ fail to apply to (5). On the other hand, (5) cannot be reduced to a form in which some other known results may be used.

We could establish corresponding theorems by the method that is used in this note, which would improve other results of [8].

ACKNOWLEDGEMENTS. I would like to thank the referee for his valuable comments. I would also like to thank Professor George W. Johnson for his generous help during my stay at the University of South Carolina. 


\section{REFERENCES}

1. F. V. Atkinson, On second order nonlinear oscillations, Pacific J. Math. 5 (1955), 643-647.

2. W. J. Coles, An oscillation criterion for second-order differential equations, Proc. Amer. Math. Soc. 19 (1968), 755-759.

3. P. Hartman, On nonoscillatory linear differential equations of second order, Amer. J. Math. 74 (1952), 389-400.

4. I. V. Kamenev, Integral criterion for oscillations of linear differential equations of second order, Mat. Zametki 23 (1978), 249-251.

5. D. Willett, On the oscillatory behavior of the solutions of second order linear differential equations, Ann. Polon. Math. 21 (1969), 175-194.

6. A. Wintner, A criterion of oscillatory stability, Quart. Appl. Math. 7 (1949), 115-117.

7. C. C. Yeh, An oscillation criterion for second order nonlinear differential equations with functional arguments, J. Math. Anal. Appl. 76 (1980), 72-76.

8. Oscillation theorems for nonlinear second order differential equations with damped term, Proc. Amer. Math. Soc. 84 (1982), 397-402.

Department of Mathematics, Shanxi University, Taiyuan, Shanxi, People's Republic of China (Current address)

Department of Mathematics and Statistics, University of South Carolina, Columbia, South Carolina 29208 